\title{
Frequent Placental SARS-CoV-2 in Patients with COVID-19-Associated Hypertensive Disorders of Pregnancy
}

\author{
Marta Fabre ${ }^{a, b}$ Pilar Calvo ${ }^{b, c}$ Sara Ruiz-Martinez ${ }^{b, c, d}$ Maria Peran ${ }^{a, b}$ Daniel Oros ${ }^{b, c, d}$ \\ Ana Medel-Martinez ${ }^{e}$ Mark Strunk ${ }^{e}{ }^{f}$ Rafael Benito Ruesca ${ }^{g}$ Jon Schoorlemmer ${ }^{b}, h, i$ \\ Cristina Paules ${ }^{b, c, d}$
}

anstituto de Investigación Sanitario de Aragón (IIS Aragon) Biochemistry Department, Hospital Clínico Universitario Lozano Blesa Zaragoza, Zaragoza, Spain; bPlacental Pathophysiology \& Fetal Programming Research Group, B46_20R \& GIIS-028 Del IISA, Instituto de Investigación Sanitario de Aragón (IIS Aragon), Zaragoza, Spain; CInstituto de Investigación Sanitario de Aragón (IIS Aragon), Obstetrics Department, Hospital Clínico Universitario Lozano

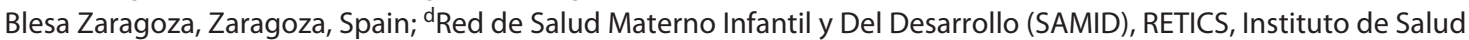
Carlos III (ISCIII), Subdirección General de Evaluación y Fomento de La Investigación y Fondo Europeo de Desarrollo Regional (FEDER) Ref: RD16/0022/0013, Zaragoza, Spain; 'Laboratorio Satélite, Instituto Aragonés de Ciencias de La Salud (IACS), Centro de Investigación Biomédica de Aragón (CIBA), Zaragoza, Spain; ‘Sequencing and Functional Genomics, Instituto Aragonés de Ciencias de La Salud (IACS), Centro de Investigación Biomédica de Aragón (CIBA), Zaragoza, Spain; ${ }^{9}$ Microbiology Department, Hospital Clínico Universitario Lozano Blesa, Zaragoza, Spain, Universidad de Zaragoza, IIS Aragon, Zaragoza, Spain; ' Instituto Aragonés de Ciencias de La Salud (IACS), Zaragoza, Spain; 'ARAID Foundation, Zaragoza, Spain

\section{Mini Summary}

- What does this study add to current knowledge? (80 words max.)-Our study shows that among women diagnosed with COVID-19 during pregnancy, the presence of SARS-CoV-2 in the placenta was more frequent among women suffering from preeclampsia or gestational hypertension. Moreover, we find that more severe hypertensive disorders were associated with higher viral load in placenta. We identify several cases of placental SARS-CoV-2 positivity at delivery (RT-PCR) in women who have tested negative for SARS-CoV-2 after an initial COVID-19 episode.

- What are the main clinical implications? (80 words max.)-SARS-CoV-2 infection could trigger pathophysiological pathways that could favor the development of hypertensive disorders. The presence of viral load in the placenta weeks after the infection, accompanied by hypertensive disorders, could be considered as a form of chronic COVID-19. We should take this information into account when controlling pregnant women after SARS-CoV-2 infection.

\section{Keywords}

SARS-CoV-2 - Placenta $\cdot$ Hypertensive disorders of pregnancy $\cdot$ COVID-19. Viral load

\begin{abstract}
Introduction: Studies described an increased frequency of hypertensive disorders of pregnancy (HDP) after a COVID-19 episode. There is limited evidence about SARS-CoV-2 viral load in placenta. This study aimed to investigate the relationship between SARS-CoV-2 viral load in the placenta and clin-
\end{abstract}

Correspondence to:

Jon Schoorlemmer, jonas@ araid.es 
ical development of HDP after COVID-19 throughout different periods of gestation. Methods: This is a case-control study in women with and without gestational hypertensive disorders after SARS-CoV-2 infection diagnosed by RT-PCR during pregnancy. Patients were matched by gestational age at the moment of COVID-19 diagnosis. We performed an analysis of SARS-CoV-2 RNA levels in placenta. Results: A total of 28 women were enrolled. Sixteen patients were diagnosed with COVID-19 during the third trimester and the remaining 12 patients in the other trimesters. Ten placentas (35.7\%) were positive for SARS-CoV-2, 9 of them (9/14,64.3\%) belonged to the HDP group versus $1(1 / 14,7.2 \%)$ in the control group $(p=0.009)$. Those cases with the highest loads of viral RNA developed severe preeclampsia (PE). Conclusion: Among women diagnosed with COVID-19 during pregnan$\mathrm{cy}$, the presence of SARS-CoV-2 in the placenta was more frequent among women suffering from $\mathrm{PE}$ or gestational hypertension. Furthermore, the most severe cases of HDP were associated with high placental viral load, not necessarily associated with a positive nasopharyngeal RT-PCR at delivery. Our data suggest that SARS-CoV-2 infection during pregnancy could trigger gestational hypertensive disorders through persistent placental infection and resulting placental damage.

(c) 2021 S. Karger AG, Basel

\section{Introduction}

Several international studies have recently suggested an increase in preeclampsia (PE) and other hypertensive disorders of pregnancy (HDP) associated with SARSCoV-2 infection during pregnancy [1-4] with classic signs and symptoms related to PE, such as hypertension, proteinuria, thrombocytopenia, and elevated liver enzymes $[5,6]$. PE is a pregnancy-specific multisystem disorder characterized by endothelial dysfunction that leads to the release of soluble factors into de maternal circulation responsible for the multi-organ injury [7]. These socalled PE-like syndromes are in some way clinical manifestations of maternal endothelial dysfunction.

The clinical manifestations of the SARS-CoV-2 infection support a key role for endothelial dysfunction in the pathobiology of this condition. SARS-CoV-2 infection induces an endotheliitis in different organs as a direct consequence of viral infection and the host inflammatory response $[8,9]$. Angiotensin-converting enzyme 2 is the major cellular-entry receptor for SARS-CoV-2 virus [10]. Angiotensin-converting enzyme 2 is present prominently in the alveoli, but also in endothelial cells, which can be directly infected by the virus [11]. Besides, cytokine release syndrome can also drive endothelial damage independently [12]. Consequently, a distinctive feature of SARS-CoV-2 infection is vascular harm, with severe endothelial injury, widespread thrombosis, and microangiopathy, in response to endothelial damage. Therefore, endothelial dysfunction seems to be the pathophysiological substrate for severe COVID-19 complications.

It is at present unknown how COVID-19 is associated with increased frequency of HDP (and especially PE) and whether similar mechanisms operate. COVID-19-associated PE-like disease may be an indirect result of maternal disease or more directly related to the presence of SARS$\mathrm{CoV}-2$ in the placenta. While initial studies had suggested that SARS-CoV-2 invades the placenta in some cases [13$15]$, posterior data suggest that it is not very common [16-19]. Consistent with a low frequent presence of SARS-CoV-2 in the placenta [20], vertical transmission is rather infrequent [21]; a systematic review reported a rate of $3.2 \%$ vertical transmission in mothers who tested positive for SARS-CoV-2 (diagnosed by RT-PCR) during the third trimester of pregnancy [22]. A recent study of placentas taken from COVID-19-positive mothers [23] describes a low-level presence of RNA in about half of placentas. No substantial histopathological, maternal, or neonatal outcome features were detected associated with this low level of viral RNA. However, a high SARS-CoV-2 RNA level was detected in only one patient, who exhibited severe placental injury in the form of extensive fibrin deposition, necrosis of the syncytiotrophoblast layer of the villi and apoptosis. This observation fits the hypothesis that only limited placental pathology is associated with COVID-19, except for cases with very high SARSCoV-2 RNA levels, the latter indicative of high viral load.

Considering the increase in hypertensive disorders in COVID-19 patients, this study aimed to investigate the relationship between SARS-CoV-2 viral load in placental tissue at birth and the development of PE or gestational hypertension after COVID-19 throughout different periods of gestation. The clinical history of 3 cases of PE, with positive placental RT-PCR tests for SARS-CoV-2 during each of the 3 semesters of pregnancy, is described in detail as an example.

\section{Materials and Methods}

We conducted a case-control study in women diagnosed with COVID-19 during pregnancy with and without gestational hypertensive disorders. We performed an analysis of SARS-CoV-2 RNA levels in placental tissue and SARS-CoV-2 antibodies in the um- 
bilical cord. Patients were recruited at the moment of delivery between May 2020 and February 2021 at a tertiary university center. Cases were defined as women who tested positive for SARS-CoV-2 during pregnancy and were diagnosed with gestational hypertensive disorders. Controls, defined as SARS-CoV-2-positive women without HDP during pregnancy, were paired by gestational age at the moment of SARS-CoV-2 diagnosis.

SARS-CoV-2 infection was diagnosed based on the positive RT-PCR test for SARS-CoV-2 from nasopharyngeal swabs. RTPCR test kits from different companies were used: Viasure (CerTest Biotec, Zaragoza, Spain), M2000 SARS-CoV-2 Assay (Abbott RealTime SARS-CoV-2 Assay, Abbott Molecular, Abbott Park, IL, USA), TaqPath COVID-19 (Thermo Fisher Scientific, USA-FDA) and Alinity SARS-CoV-2 (Abbott Alinity, Abbott Molecular, Abbott Park, IL, USA). Information on these test kits are listed in online supplementary Table 1 (for all online suppl. material, see www.karger.com/doi/10.1159/000520179). As suggested by the manufacturer for nasopharyngeal specimens, cycle threshold (CT) values below 37 were taken as positive. Besides, all women who were hospitalized for delivery were screened for SARS-CoV-2 infection using RT-PCR on nasopharyngeal swabs shortly before giving birth. However, in the case of pregnant women who had already overcome the infection, RT-PCR was only performed around delivery if the latest negative RT-PCR test was more than 3 months ago. As a result, 4 patients $(3,4,15$, and 16) were not tested for SARS-CoV-2 infection at the time of delivery. Prophylactic treatment with low molecular weight heparin for 14 days was applied to COVID-19 women diagnosed by RT-PCR [24]. COVID-19 has been divided into 3 types [25]: asymptomatic infection refers no clinical symptoms or signs; mild infection refers to symptoms such as fever, cough, headache, anosmia and asthenia; and severe infection refers dyspnea, hypoxemia accompanied by chest imaging compatible with pneumonia and respiratory infection.

Placental tissue samples were taken at the moment of delivery, taking villous tissue while carefully trying to avoid the overlying maternal tissue. Placental samples, approximately $1 \mathrm{~cm}^{3}$ in size, were placed into tubes containing $1 \mathrm{~mL}$ of preservative solution (RNAlater Fisher Scientific) at $4^{\circ} \mathrm{C}$. After $24 \mathrm{~h}$, excess RNAlater was removed, and the samples were stored at $-80^{\circ} \mathrm{C}$. Tissue was homogenized; RNA was extracted, treated with DNase I, repurified and resuspended as previously described [26]. Concentrations were measured using Nanodrop, 100 ng of RNA was used in downstream RT-PCR analysis. RT-PCR for SARS-CoV-2 in placenta tissue samples was performed using the TaqPath COVID-19 CEIVD RT-PCR kit (Catalog number A48067; Life Technologies Europe, Bleiswijk, The Netherlands) on a QuantStudio5 (Applied Biosystems; Thermo Fisher Scientific) apparatus. The limit of detection of this kit is listed as 10 genomic copy equivalents (GCEs). As this assay targets N, ORF1aboratory and S genes, only samples with 3 positive targets were considered as SARS-CoV-2 positive. SARS-CoV-2 tissue load in the placenta is referred to in the manuscript as viral load and is expressed as CT values of RT-PCR [27].

We collected umbilical cord plasma in EDTA and samples were centrifuged. Serological assays were determined on the same day by chemiluminescent microparticle immunoassays for quantitative detection of SARS-CoV-2 IgG. The SARS-CoV-2 IgG assay is designed to detect IgG antibodies to the nucleocapsid protein of SARS-CoV-2 (IgG; SARS-CoV-2 IgG Assay, Abbott Laboratories Ireland, Dublin, Ireland) and IgM + IgA antibodies (IgM + IgA; COVID-19 VIRCLIA IgM + IgA, Vircell Microbiologists, Granada, Spain).

SARS-CoV-2 Viral Load in the Placenta of Patients with Hypertensive Disorders
Gestational hypertensive disorders were divided into 4 categories, defined according to the criteria proposed by American College of Obstetrics and Gynecologists [28]: PE, chronic hypertension, chronic hypertension with superimposed $\mathrm{PE}$ and gestational hypertension. First trimester risk of early-onset PE was retrospectively calculated according to maternal characteristics, obstetric history, maternal blood pressure, maternal serum pregnancy-associated plasma protein-A, and uterine artery pulsatility index, using SsdwLab6 version 6.1 package (SBP Soft 2007 S.L.) [29].

Clinical characteristics, laboratory results, and maternal and neonatal outcomes were collected from medical records. All patients provided written informed consent. The study was approved by the Research Ethics Committee of the Community of Aragon (C.I. PI21/155 and COL21/000) and all patients provided written informed consent.

Statistical analysis was performed using SPSS 22.0. Categorical variables were presented as frequencies or percentages. Continuous variables were presented using mean \pm standard deviation (SD), median, or range. For continuous variables, Shapiro-Wilk tests of normality were used to evaluate the distributions. Data were analyzed using the Student $t$ test or Mann-Whitney U test. Statistical significance was considered $p<0.05$.

\section{Results}

Fourteen women in the case group (COVID-19 positive and diagnosis of HDP) and 14 women in the control group, matched for gestational age at the time of $\mathrm{CO}$ VID-19 diagnosis, were included. Out of those 14 cases, 9 cases $(64.3 \%)$ had PE and 5 cases (35.7\%) had gestational hypertension. Demographic and clinical data, as well as perinatal and neonatal outcomes, are shown in Table 1. There were no significant differences in maternal age, maternal weight, risk of $\mathrm{PE}$ at $1^{\circ}$ trimester, nulliparity rate, and diabetes mellitus between the 2 groups. The vast majority of patients, 8 pairs, represent pregnant women who were diagnosed with COVID-19 during the third trimester, whereas 5 pairs in the second trimester and only 1 pair in the first trimester. The mean gestational age at delivery was 268.1 days in the case group and 278.9 days in the control group $(p=0.005)$. Women with HPD also exhibited lower birth weight than those in the control group $(2,852.5$ g SD 467.0 vs. $3,282.9$ g SD: 435.9 ; $p=0.005)$. There were no significant differences for small for gestational age, umbilical arteria ph $<7$.1. Induced labor and caesarean were significantly more frequent in the HDP group than in controls $(p=0.003$ and $p=0.015$, respectively).

The chronology of diagnosis of HDP and SARS-CoV-2 infection of each patient is summarized in Table 2. There was no significant difference in the severity of initial SARS-CoV-2 symptoms between the groups $(p=0.341)$.

Fetal Diagn Ther 2021;48:801-811 803 
Table 1. Demographic data and obstetrical and SARS-CoV-2 infection characteristics

\begin{tabular}{|c|c|c|c|}
\hline & $\begin{array}{l}\text { Case group } \\
(n=14)\end{array}$ & $\begin{array}{l}\text { Control group } \\
(n=14)\end{array}$ & $p$ value \\
\hline \multicolumn{4}{|l|}{ Demographic maternal data } \\
\hline Maternal age (SD), years & $31.93(6.6)$ & $30.36(5.8)$ & 0.506 \\
\hline Maternal weight (SD), kg & $69.32(10.5)$ & $68.57(13.2)$ & 0.868 \\
\hline Caucasian, \% & $9(64.3)$ & $4(28.6)$ & 0.063 \\
\hline $1 \circ$ trimester high risk for $\mathrm{PE}, \%$ & $5(35.7)$ & $3(21.4)$ & 0.194 \\
\hline Diabetes mellitus, $\%$ & $0(0)$ & $1(7.14)$ & 0.317 \\
\hline Nulliparous, $\%$ & $4(28.6)$ & $7(50)$ & 0.317 \\
\hline \multicolumn{4}{|l|}{ Maternal and neonatal outcome at delivery } \\
\hline Gestational age at birth mean (SD), days & $268.1(10.8)$ & $278.9(7.8)$ & 0.005 \\
\hline Birth weight, mean (SD), kg & $2,852.5(467.0)$ & $3,282.9(435.9)$ & 0.018 \\
\hline SGA (<10th percentile), \% & $4(28.6)$ & $1(7.2)$ & 0.146 \\
\hline Induced labor, \% & $12(85.1)$ & $4(28.6)$ & 0.003 \\
\hline Caesarean delivery, $\%$ & $5(35.7)$ & $0(0)$ & 0.015 \\
\hline $\mathrm{pH}<7.1, \%$ & $2(14.3)$ & $0(0)$ & 0.541 \\
\hline Pre-term birth (<37 wk gestation), $\%$ & $3(21.4)$ & $0(0)$ & 0.072 \\
\hline \multicolumn{4}{|l|}{ Description of SARS-CoV-2 } \\
\hline \multicolumn{4}{|l|}{ Trimester of SARS-CoV-2 infection } \\
\hline $10, \%$ & $1(7.2)$ & $1(7.2)$ & \\
\hline $2^{\circ}, \%$ & $5(35.7)$ & $5(35.7)$ & \\
\hline $30, \%$ & $8(57.1)$ & $8(57.1)$ & 1 \\
\hline \multicolumn{4}{|l|}{ COVID-19 symptoms } \\
\hline No & $6(42.9)$ & $7(50)$ & \\
\hline Mild & $6(42.9)$ & $7(50)$ & \\
\hline Severe & $2(14.2)$ & $0(0)$ & 0.341 \\
\hline Interval from diagnosis of SARS-CoV-2 to delivery (SD), d & $61.86(54.79)$ & $72.64(57.7)$ & 0.616 \\
\hline Positive SARS-CoV-2 RT-PCR placenta tissue $(C T<37), \%$ & $9(64.3)$ & $1(7.2)$ & 0.009 \\
\hline Neonatal lgG, $* \%$ & $6(42.9)$ & $7(50)$ & 0.688 \\
\hline
\end{tabular}

SD, standard deviation; CT, cycle threshold value RT-PCR; SGA, small for gestational age; PE, preeclampsia. * Analyzed 24 of 28.

Thirteen had no symptoms, 13 patients had mild and only 2 patients had severe SARS-CoV-2 infection (Table 2). No patient required admission to the intensive care unit.

We analyzed placentas delivered by 28 women who had tested positive for SARS-CoV-2 at some point during pregnancy using a sensitive RT-PCR assay. Ten placentas (35.7\%) tested positive for SARS-CoV-2 RNA at birth. In the case group, we found 9 positive placentas $(9 / 14$, $64.3 \%)$ while in the control group only 1 placenta $(1 / 14$, $7.2 \%)$ was positive $(p=0.009)$ (shown in Fig. 1$)$. Moreover, 7 of 9 placentas $(77.8 \%)$ belong to women diagnosed with PE. Focusing on positive placentas, we have 3 cases with high loads of viral RNA (CTs $\leq 28$ ) (see below), and the remaining 7 cases with low loads of viral RNA (median CT of 30.6; interquartile range 28.7-33.9) (Table 2).

While only 6 pairs of pregnancies with positive SARS$\mathrm{CoV}-2$ tests during the first or the second trimester (GA $\leq 27$ weeks) were analyzed (Table 2 ), several remarkable results deserve mentioning. First, out of the 6 cases with HDP, 6 out of 6 had a positive placental SARS-CoV- 2 test at birth. In contrast, among the corresponding 6 control cases without HDP, only 1 tested positive for placental SARS-CoV-2 (Table 2). It is possible therefore that the continued presence of SARS-CoV-2 in the placenta promotes HDP. Moreover, out of 10 pregnancies with these early SARS-CoV-2 infections for which data are available, only 1 mother remained positive at delivery ( $\mathrm{N}^{\circ} 6$ ), while 7 placentas tested positive. This latter data suggest that infection with SARS-CoV-2 during early pregnancy may commonly result in persisting placental presence of SARS-CoV-2.

Finally, antibody quantification was performed for $87.5 \%$ neonates with umbilical cord blood samples. Thirteen infants tested positive for IgG on the first 10 days of life, and none tested positive for IgM + IgA. There was no significant difference between the groups in the number 


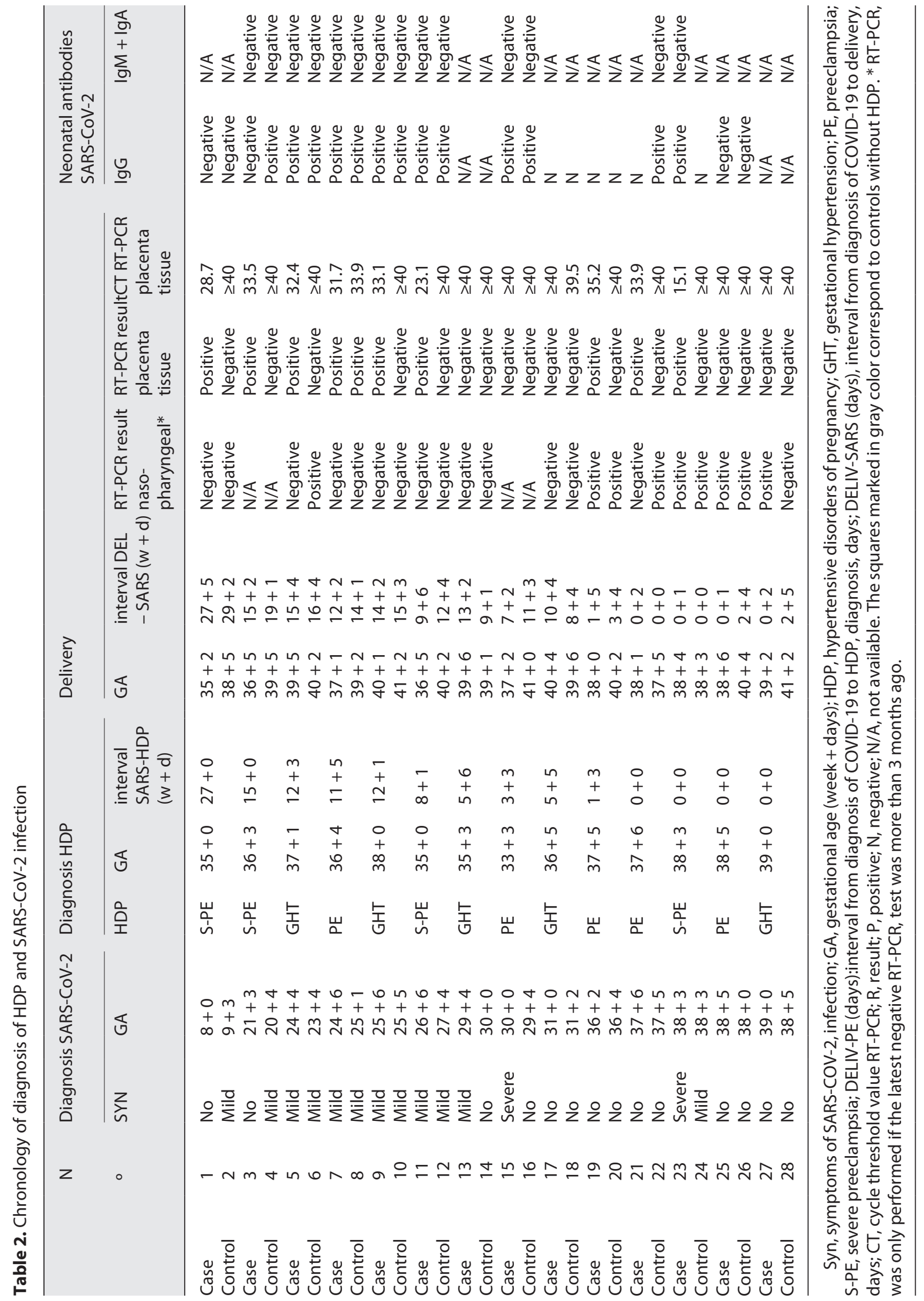


Fig. 1. \% RT-PCR-positive placenta: case group versus control group. $\mathrm{N}$ total $=28$. case group $=14$ and control group $=14$. Ten placentas $(35.7 \%=3.57 \%+32.14 \%)$ tested positive for SARS-CoV-2 RNA. Nine placentas were positive in the case group $(9 / 28,32.14 \%)$, while in the control group, only 1 placenta was positive $(1 / 28,3.57 \%)$. Seven $(7 / 28,25 \%)$ belong to women diagnosed with PE versus 2 of 9 belong to women diagnosed with gestational hypertension $(2 / 28,7.14 \%)$. PE, preeclampsia; LMWH, low molecular weight heparin; IUGR, intrauterine growth restriction; CT, cycle threshold.

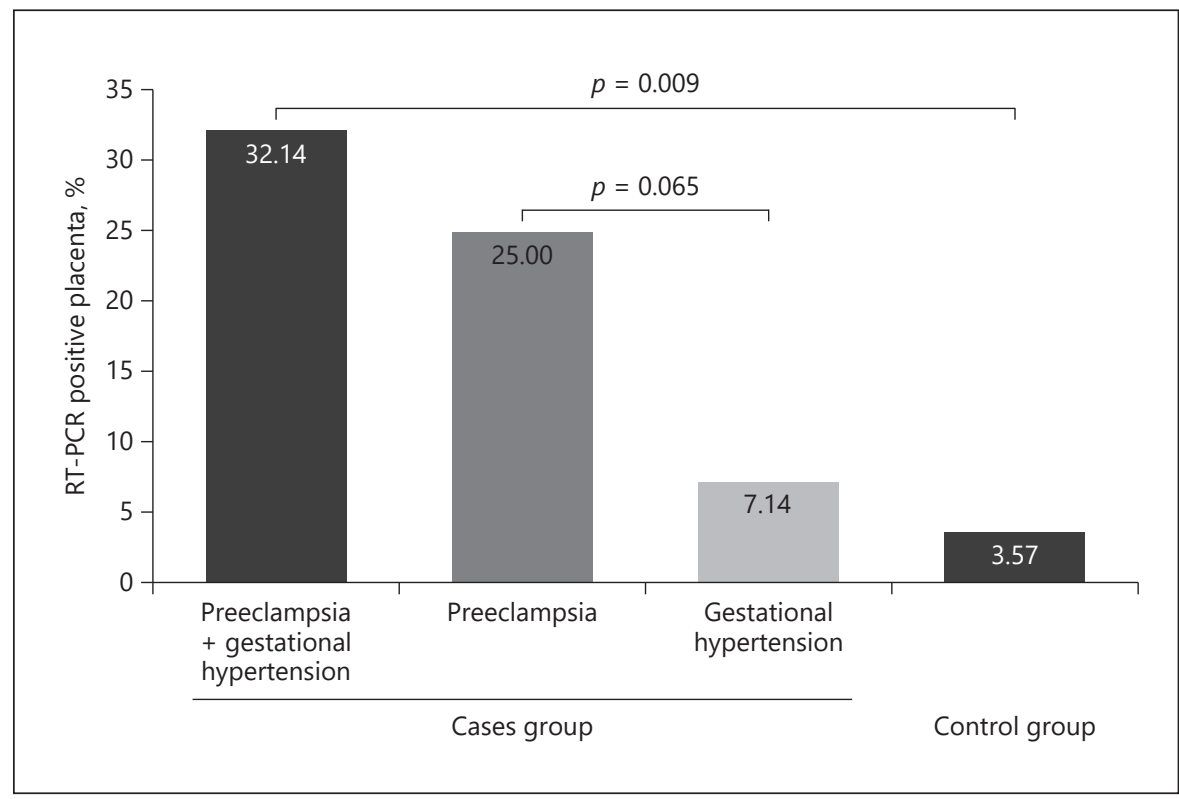

\section{Case 1}

1st Trimester
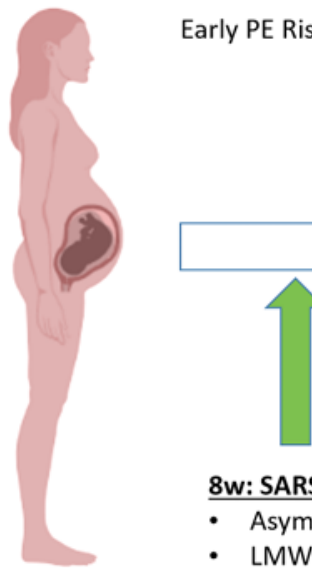

Early PE Risk: 1/122

8w: SARS-CoV-2 RT-PCR positive

- Asymptomatic

- LMWH $\times 14$ days 3rd Trimester:

EFW: 2045gr IUGR (P1)

\section{5+2w: Delivery}

Induction : Instrumental Delivery SARS-CoV-2 RT-PCR: negative

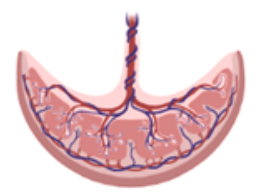

Placenta SARS-CoV-2 test: positive CT: 28.716

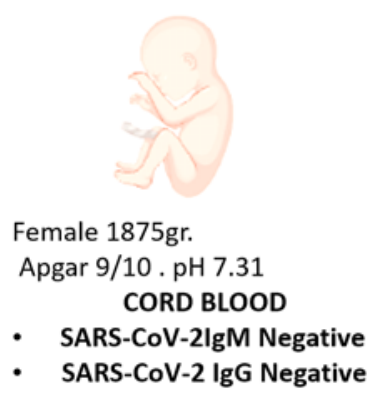

Fig. 2. Time line of case 1. TPAL, term, pre-term, abortion, life; LMWH, low molecular weight heparin; PAPP-A, pregnancy-associated plasma protein-A; MoM, multiple of median; BP, mean arterial blood pressure; UtA PI, uterine artery resistance pulsatility index; w, weeks; EFW, estimated fetal weight; PI UA, umbilical artery pulsatility index; PI MCA: fetal middle cerebral artery pulsatility index; IUGR, intrauterine growth restriction; BP, blood pressure; CT, cycle threshold. of neonates that tested positive for SARS-CoV-2 IgG $(p=$ 0.688).

Among the placentas that tested positive, 3 cases with high loads of viral RNA (CTs $=15 ; 23 ; 28$, respectively) represented cases of severe $\mathrm{PE}$ (Table 2). Case number 1 (shown in Fig. 2) was a woman with no medical history of interest. The estimated risk of early-onset PE in the first trimester was $1 / 122$. She presented positive SARS-CoV-2 


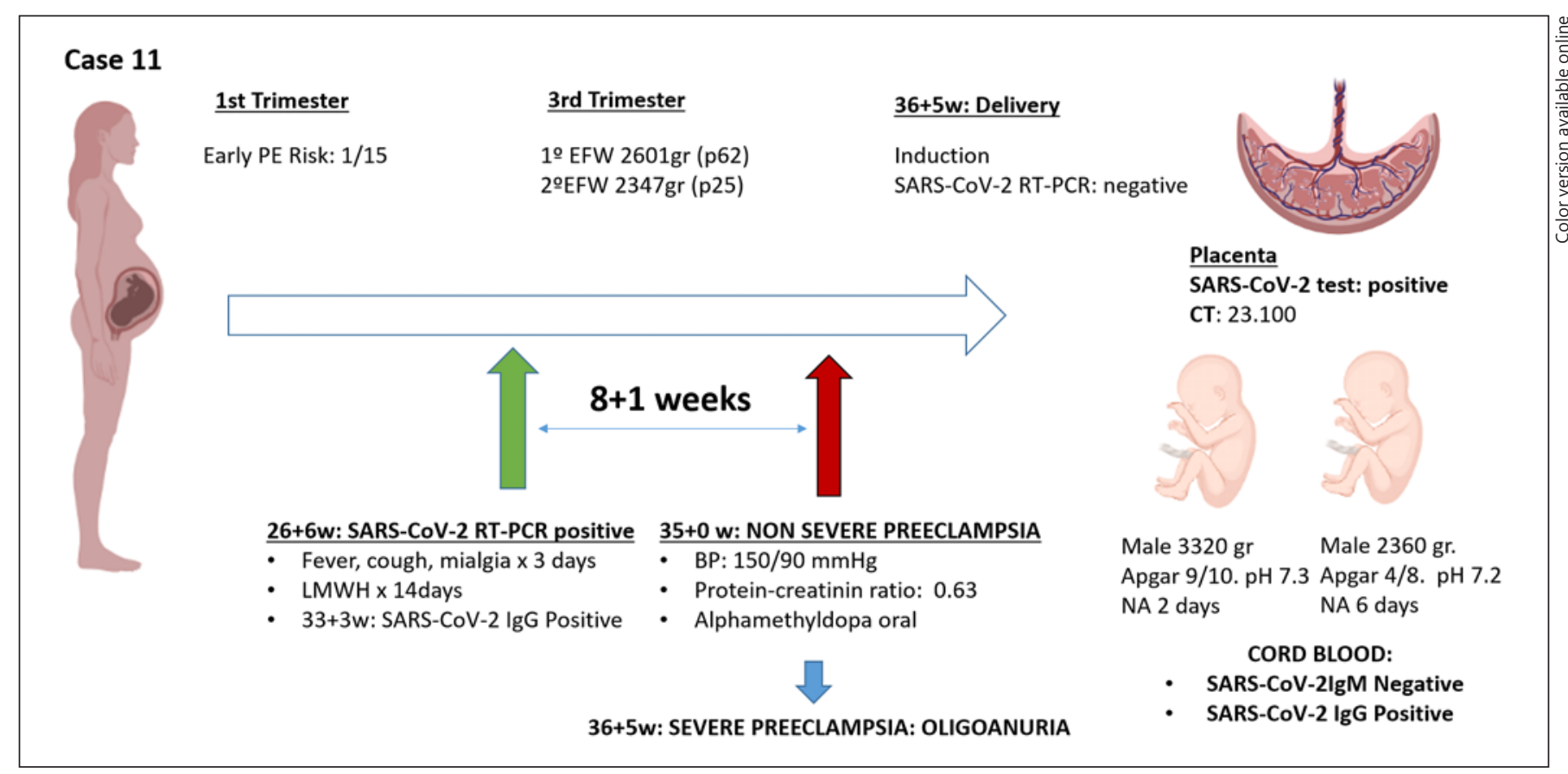

Fig. 3. Time line of case 11. TPAL, term, pre-term, abortion, life; LMWH, low molecular weight heparin; PAPP-A, pregnancy-associated plasma protein-A; MoM, multiple of median; BP, mean arterial blood pressure; UtA PI, uterine artery resistance pulsatility index; w, weeks; EFW, estimated fetal weight; PI UA, umbilical artery pulsatility index; PI MCA, fetal middle cerebral artery pulsatility index; IUGR, intrauterine growth restriction; BP, blood pressure; NA, neonatal admission; PE, preeclampsia; CT, cycle threshold.

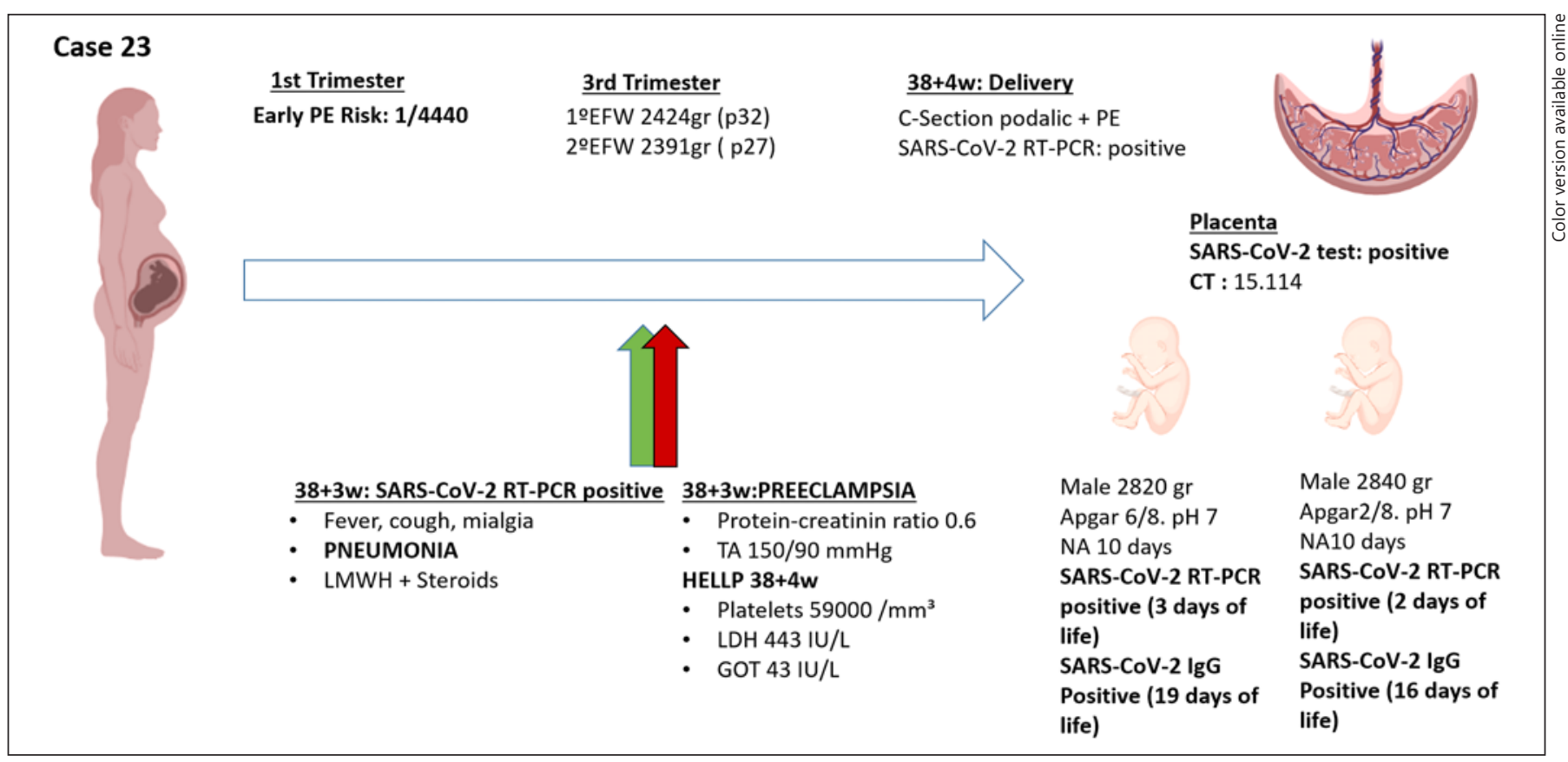

Fig. 4. Time line of case 23. TPAL, term, pre-term, abortion, life; LMWH, low molecular weight heparin; PAPP-A, pregnancy-associated plasma protein-A; MoM, multiple of median; BP, mean arterial blood pressure; UtA PI, uterine artery resistance pulsatility index; w, weeks; EFW, estimated fetal weight; PI UA, umbilical artery pulsatility index; PI MCA, fetal middle cerebral artery pulsatility index; IUGR, Intrauterine growth restriction; BP, blood pressure; NA, neonatal admission; PE, preeclampsia; CT, cycle threshold.
SARS-CoV-2 Viral Load in the Placenta of Patients with Hypertensive Disorders 
RT-PCR (CT: 18.7) at week 8 of gestation and infection courses asymptomatic. Two weeks later, she tested negative for SARS-CoV-2 by RT-PCR. At 35 week of gestation, she debuted with severe $\mathrm{PE}$, which required admission to the hospital and treatment with intravenous labetalol and magnesium sulfate. RT-PCR for SARS-CoV-2 infection was performed at the time of admission, with a negative result. Termination of pregnancy was in week $35+2$ for this reason. A female of $1,875 \mathrm{~g}$, APGAR test was 9 and 10 at 1 and $5 \mathrm{~min}$, respectively, and $\mathrm{pH} 7.31$ without antibodies against SARS-CoV-2 was born. Placental analysis for SARS-CoV-2 was positive, with a high viral load by RT-PCR.

Case number 11 (shown in Fig. 3) was a nulliparous woman with a spontaneous monochorionic diamniotic twin. The estimated risk of early-onset PE in the first trimester was $1 / 15$. Pregnancy of normal course until week $26+5$, when she debuted with fever, cough, and myalgias. RT-PCR results for SARS-CoV-2 was positive (CT: 5.9). At $34+0$ she tested negative for SARS-CoV-2 by using RT-PCR. In week 35, the patient presented nonsevere PE, well controlled with oral treatment with alfamethyldopa. At $36+5$ weeks was decided an elective delivery due to the onset of severe PE, ending in an intrapartum caesarean section. The first male twin was born: $3,320 \mathrm{~g}$, the APGAR test was 9 and 10 at 1 and 5 min, respectively, and was admitted for observation for 2 days. Second twin, male, 2,360 g, with the APGAR test of 4 and 8 at 1 and 5 $\mathrm{min}$, and he was admitted for observation for 6 days. Cord blood serology was positive for IgG and negative for IgM. The placenta was clearly positive for SARS-CoV 19 by using RT-PCR.

Case 23 (shown in Fig. 4) was a woman with a bi-chorionic biamniotic twin pregnancy. The estimated risk of early-onset $\mathrm{PE}$ in the first trimester was $1 / 4,440$. Gestation of normal course until onset of fever, cough, and malaise, with positive result in RT-PCR SARS-CoV-2 (CT: 20.2$)$ at $38+3$ weeks of gestation. The patient presented blood pressure around 150/90 $\mathrm{mm} \mathrm{Hg}$, with positive creatinine protein ratio and pneumonia on chest Xray that required treatment with low molecular weight heparin, steroids, and oxygen therapy. Laboratory tests were performed in week $38+4$, HELLP syndrome was detected $\left(59,000 \mathrm{~mm}^{3}\right.$ platelets, LDH $443 \mathrm{U} / \mathrm{L}$ and GOT $43 \mathrm{U} / \mathrm{L}$ ), and it was decided to elective delivery at that time by caesarean section due to HELLP and breech presentation. The birth weight of the first male twin was $2,820 \mathrm{~g}$, with the APGAR test 6 and 8 at 1 and $5 \mathrm{~min}$, respectively. The birth weight of the second male twin was $2,840 \mathrm{~g}$, the APGAR test was 2 and 8 at 1 and $5 \mathrm{~min}$, re- spectively. Both were admitted to the neonatal unit and the mother had no contact with the newborns after the caesarean section. RT-PCR SARS-CoV-2 was performed on both, positive at 3 and 2 days of life, respectively, as well as SARS-CoV-2 serologies, which were positive at 19 and 16 days of life, respectively. The analysis of the placenta yielded a clear positive result for SARS-CoV-2 (CT 15.1) [6].

\section{Discussion/Conclusion}

Placental histopathology at term in women suffering from COVID-19 has been examined in relation to disease symptoms such as systemic inflammatory responses, hypercoagulation, and intervillous thrombi $[30,31]$. The presence of SARS-CoV-2 in the placenta has been studied in the context of horizontal transmission of the virus [22] with an early study reporting $7.7 \%$ positivity $(2 / 26)$. However, only a very limited number of studies report data that allow a direct comparison between the placental presence of SARS-CoV-2 and HDP. An early prospective cohort study [16] describes the inability to detect SARS$\mathrm{CoV}-2$ in the placenta by RNA in situ hybridization in 44 women with SARS-CoV-2 diagnosis in the third trimester. Afterward, since the first report describing the detection of SARS-CoV-2 fetal membrane samples [32], similar reports have followed. Hosier et al. [33] mention high viral load in the placenta $(\mathrm{CT}<16)$ of a single woman with severe PE and SARS-CoV-2 infection during the late second trimester of pregnancy. In a posterior study, a rate of 47\% SARS-CoV-2-positive placenta tissue was reported in 21 women who were diagnosed (using RT-PCR) between 35 and 40 weeks of gestation [23]. In this latter study, a woman who exhibited severe placental damage delivered a baby with neurological manifestations. Based on these combined 2 cases, the possibility was raised that the severity of SARS-CoV-2-mediated placental pathology might be directly related to placental SARS-CoV-2 viral load.

Following up on these studies, we further assessed the relationship between the SARS-CoV-2 viral load in placental tissue and the development of HDP in $14 \mathrm{HDP}$ cases and paired controls, all diagnosed with COVID-19. Out of 28 samples tested in our study, we identified 10 placental tissue samples that tested positive for SARSCoV-2 (35.7\% of total), 9 of them belonging to the HDP group. We observed that, among patients with SARS$\mathrm{CoV}-2$ infection during gestation, the frequency of a positive placental SARS-CoV-2 RT-PCR test was much high- 
er in the HDP group than the non-HDP group. Furthermore, we detected that the percentage of cases with a positive placental SARS-CoV-2 RT-PCR test trend differently between PE and AHT $(p=0.065)$. In fact, the 3 most severe cases of PE (cases No 1, 11 and 23), showed higher placental viral load than all the others. Moreover, each of the 3 tested positive for SARS-CoV-2 in a different trimester of gestation. It has been argued that localization of SARS-CoV-2 to fetal cells such as the syncytiotrophoblast as opposed to maternal uterine cells requires detection by immunohistochemistry or RNA in situ hybridization [16]. We only applied RT-PCR and can therefore not exclude that the placenta samples we have analyzed do contain maternal tissue. Considering that maternal tissue was avoided when taking samples, and for the sake of simplicity, we interpret positive placental RT-PCR results as representative of viral load in villous placenta. This might indicate that in women susceptible to HDP, the presence of placental SARS-CoV-2 may contribute to the severity of the disorder. We suggest that a relationship between a higher viral load in placenta tissue and the severity of the hypertensive disorder deserves consideration.

Placental characteristics described in SARS-CoV-2 infection include maternal vascular perfusion and inflammation [30, 34]. It has been postulated that the detrimental health outcomes associated with HDP are the result of generalized endothelial and vascular dysfunction [35]. Similarly, a consequence of SARS-CoV-2 infection is endothelial injury in different organs resulting directly or indirectly from the host inflammatory response [9]. Alternatively [36], it has been proposed that direct infection of syncytioprophoblast cells in the placenta by SARSCoV-2 may cause placental dysfunction and pregnancy complications [37]. The pathophysiological mechanisms that connect placental SARS-CoV-2 with HDP remain to be established.

The main limitation of our study is the small sample size. Further studies with a large number of patients are required to confirm the association between high SARSCoV-2 viral load in placenta tissue and the development of HDP. Moreover, our hospital's protocols changed as the pandemic progressed. Therefore, different methodologies have been used for viral RNA detection. As not all tests proved to be equally sensitive and specific, the use of distinct tests may have caused a discrepancy with studies published previously $[13,16,22]$. In addition, we do not have sequencing data from nasopharyngeal maternal swabs available, but all pregnant women were diagnosed before the alpha (B.1.1.7 UK) variant and delta (B.1.617.2 India) were routinely detected in our area. A final limita-

SARS-CoV-2 Viral Load in the Placenta of Patients with Hypertensive Disorders tion to address is the fact that for each placenta, we tested only a single sample. As SARS-CoV-2 is not necessarily homogenously present (or absent) throughout the placenta, the identification of samples as positive or negative should be interpreted in this context.

Among the strengths of the study, we highlight the inclusion of women who tested positive for SARS-CoV-2 in each of the 3 trimesters of pregnancy. We demonstrate a higher viral load in placental tissue in the case group than in the control group and a higher frequency of infected placentas. Besides, as a SARS-CoV-2 RT-PCR test carried out at the time of birth showed all mothers tested negative, we could rule out the possibility of acute maternal infection during delivery. Some cases we describe with high placenta SARS-CoV-2 levels, specifically patient 1 and patient 11, had negative RT-PCR results at the time of delivery. This data shows that placenta infection may persist in the absence of maternal infection. In the limited of cases we describe, this persistent viral presence is associated with the development of adverse perinatal outcomes.

Previous viral epidemics have confirmed that pregnant women are at increased risk for severe virus infection [38-41]. SARS-CoV-2 is not an exception, as pregnant patients with COVID-19 are at increased risk for severe illness [42] and adverse pregnancy outcomes [8]. We showed that the frequency of persistent SARS-CoV-2 in the placenta is increased among mothers with HDP, and that 6 out of 6 mothers who tested positive for SARSCoV-2 before week 27 of gestation and also showed persistent placental infection at birth developed HDP. Combined, these results suggest that chronic COVID might increase the risk of PE. We therefore suggest that healthcare professionals should monitor pregnant women after SARS-CoV-2 infections even more closely, since a possible placental infection cannot be tested until delivery.

In summary, this study assesses the relationship between SARS-CoV-2 viral load in placental tissue and the maternal risk of developing gestational hypertensive disorders. We find that the presence of SARS-CoV-2 (although we do not formally prove viral infection) was more frequent in the placentas of those in the HDP group than in the placentas of COVID-19 mothers without HDP. We detect placental RNA representative of SARS$\mathrm{CoV}-2$ infection at high levels in a limited number of $\mathrm{PE}$ cases, and at lower levels in about $25 \%$ of placentas from COVID-19 positive mothers. There are different ways in which the infant of a RT-PCR-positive mother could be affected by SARS-CoV-2: maternal inflammation and endothelial damage may be transmitted to the placenta in 
the form of cytokines which might modulate the fetal immune system [43]. Alternatively, SARS-CoV-2 infection of the placenta may directly influence the fetal environment. The presence of placental viral RNA needs to be investigated in a much larger cohort to clarify if and in what frequency if SARS-CoV-2 infection during pregnancy does trigger gestational hypertensive disorders through placenta-related mechanisms. Our work contributes to the understanding of how SARS-CoV-2 virus affects pregnancy, which includes the possibility that placental infection contributes to pregnancy complications. Better and more detailed understanding of the placental disease process may aid or could be essential for appropriate monitoring during pregnancy and providing appropriate postnatal care.

\section{Acknowledgments}

We acknowledge the use of the Sequencing and Functional Genomics Core Facility (Servicio Científico Técnico de Secuenciación y Genómica Funcional) of the Aragon Health Sciences Institute (IACS and Universidad, Zaragoza, Spain). We are grateful to all patients who provided clinical samples at the Biochemistry Department and Obstetrics Department, Hospital Clínico Universitario Lozano Blesa Zaragoza, Spain.

\section{Statement of Ethics}

The study was approved by the Research Ethics Committee of the Community of Aragon (C.I. PI21/155 and COL21/000). Written informed consent was obtained from participants.

\section{Conflict of Interest Statement}

The authors have no conflicts of interest to declare.

\section{Funding Sources}

Part of the work has been financed by research grant FIS PI17/02208 from the Instituto de Salud Carlos III to Jon Schoorlemmer. The work described in this manuscript has been financed by grants from the Government of Aragon, co-financed by FEDER ("Una manera de hacer Europa, Construyendo Europa desde Aragón"/European Social Funds): B05_17D. The funding bodies played no role in the design of the study, in the collection, analysis, and interpretation of data, in writing the manuscript, and in the decision to submit the article for publication. P Calvo, M Fabre, C Paules, D Oros, and S Ruiz-Martinez were supported by a research grant from the Instituto de Salud Carlos III (CM20/00078, FI20/00071, JR19/00006, INT20/00033, and CM18/00202).

\section{Author Contributions}

M.F., J.S., and D.O. were responsible for the design of the study and the writing of the manuscript. M.F., M.P., P.C., S.R.M., C.P., J.S., and D.O. were responsible for the analysis and interpretation of the data. PC and SRM contributed to the writing of the manuscript. M.F., M.P., M.S., A.M.M., J.S., and R.B. were responsible for the laboratory analysis. P.C., S.R.M., C.P., and D.O. were responsible for the collection of clinical data of the patients. All authors reviewed and approved the final version of the manuscript.

\section{Data Availability Statement}

The data described in this article are openly available in medRxiv https://www.medrxiv.org/content/10.1101/2021.09.07.212 $61607 \mathrm{v} 1$ ?rss=1\#disqus_thread.

\section{References}

1 Papageorghiou AT, Deruelle P, Gunier RB, Rauch S, García-May PK, Mhatre M, et al. Preeclampsia and COVID-19: results from the INTERCOVID prospective longitudinal study. Am J Obstet Gynecol. 2021 Sep;225(3): 289-e17.

2 Villar J, Ariff S, Gunier RB, Thiruvengadam R, Rauch S, Kholin A, et al. Maternal and neonatal morbidity and mortality among pregnant women with and without COVID-19 infection: the INTERCOVID Multinational Cohort Study. JAMA Pediatr. 2021 Aug 1; 175(8):817-26.

3 Di Mascio D, Khalil A, Saccone G, Rizzo G, Buca D, Liberati M, et al. Outcome of coronavirus spectrum infections (SARS, MERS, COVID-19) during pregnancy: a systematic review and meta-analysis. Am J Obstet Gynecol MFM. 2020 May;2(2):100107.
4 Ahlberg M, Neovius M, Saltvedt S, Söderling J, Pettersson K, Brandkvist C, et al. Association of SARS-CoV-2 test status and pregnancy outcomes. JAMA. 2020 Nov 3;324(17): $1782-5$.

5 Mendoza M, Garcia-Ruiz I, Maiz N, Rodo C, Garcia-Manau P, Serrano B, et al. Pre-eclampsia-like syndrome induced by severe COVID-19: a prospective observational study. BJOG. 2020;127(11):1374-80.

6 Abadía-Cuchí N, Ruiz-Martínez S, Fabre M, Mateo P, Remacha Sienes M, Ventura Faci P, et al. SARS-CoV-2 congenital infection and pre-eclampsia-like syndrome in dichorionic twins: a case report and review of the literature. Int J Gynaecol Obstet. 2021 Aug;154(2): $370-2$.
7 Romero R, Chaiworapongsa T. Preeclampsia: a link between trophoblast dysregulation and an antiangiogenic state. J Clin Invest. 2013; 123:2775-7.

8 Huertas A, Montani D, Savale L, Pichon J, Tu L, Parent F, et al. Endothelial cell dysfunction: a major player in SARS-CoV-2 infection (COVID-19)? Eur Respir J. 2020;56(1):2001634.

9 Varga Z, Flammer AJ, Steiger P, Haberecker M, Andermatt R, Zinkernagel AS, et al. Endothelial cell infection and endotheliitis in COVID-19. Lancet. 2020 May 2;395(10234): $1417-8$.

10 Zhou P, Yang XL, Wang XG, Hu B, Zhang L, Zhang W, et al. A pneumonia outbreak associated with a new coronavirus of probable bat origin. Nature. 2020;579(7798):270-3. 
11 Kumar A, Narayan RK, Kumari C, Faiq MA, Kulandhasamy M, Kant K, et al. SARS-CoV-2 cell entry receptor ACE2 mediated endothelial dysfunction leads to vascular thrombosis in COVID-19 patients. Med Hypotheses. 2020;145:110320

12 Castro P, Palomo M, Moreno-Castaño AB, Fernández S, Torramadé-Moix S, Pascual G, et al. Is the endothelium the missing link in the pathophysiology and treatment of COVID-19 complications? Cardiovasc Drugs Ther. 2021 Jun 7:1-14.

13 Schwartz DA, Morotti D, Beigi B, Moshfegh F, Zafaranloo N, Patanè L. Confirming vertical fetal infection with coronavirus disease 2019: neonatal and pathology criteria for early onset and transplacental transmission of severe acute respiratory syndrome coronavirus 2 from infected pregnant mothers. Arch Pathol Lab Med. 2020 Dec 1;144(12):1451-6.

14 Patanè L, Morotti D, Giunta MR, Sigismondi C, Piccoli MG, Frigerio L, et al. Vertical transmission of coronavirus disease 2019: severe acute respiratory syndrome coronavirus 2 RNA on the fetal side of the placenta in pregnancies with coronavirus disease 2019-positive mothers and neonates at birth. Am J Obstet Gynecol MFM. 2020 Aug;2(3):100145.

15 Kirtsman M, Diambomba Y, Poutanen SM, Malinowski AK, Vlachodimitropoulou E, Parks WT, et al. Probable congenital sarscov-2 infection in a neonate born to a woman with active sars-cov-2 infection. Cmaj. 2020; 192(24):E647-50.

16 Edlow AG, Li JZ, Collier AY, Atyeo C, James $\mathrm{KE}$, Boatin AA, et al. Assessment of maternal and neonatal SARS-CoV-2 viral load, transplacental antibody transfer, and placental pathology in pregnancies during the COVID-19 Pandemic. JAMA Netw Open. 2020;3(12): e2030455.

17 Smithgall MC, Liu-Jarin X, Hamele-Bena D, Cimic A, Mourad M, Debelenko L, et al. Third-trimester placentas of severe acute respiratory syndrome coronavirus 2 (SARS$\mathrm{CoV}-2)$-positive women: histomorphology, including viral immunohistochemistry and in-situ hybridization. Histopathology. 2020 Dec;77(6):994-9.

18 Mulvey JJ, Magro CM, Ma LX, Nuovo GJ, Baergen RN. Analysis of complement deposition and viral RNA in placentas of COVID-19 patients. Ann Diagn Pathol. 2020; 46:151530.

19 Raschetti R, Vivanti AJ, Vauloup-Fellous C, Loi B, Benachi A, De Luca D. Synthesis and systematic review of reported neonatal SARSCoV-2 infections. Nat Commun. 2020 Oct 15; 11(1):5164.
20 Yan J, Guo J, Fan C, Juan J, Yu X, Li J, et al. Coronavirus disease 2019 in pregnant women: a report based on 116 cases. Am J Obstet Gynecol. 2020;223(1):111-e14.

21 Bwire GM, Njiro BJ, Mwakawanga DL, Sabas D, Sunguya BF. Possible vertical transmission and antibodies against SARS-CoV-2 among infants born to mothers with COVID-19: a living systematic review. J Med Virol. 2021 Mar;93(3):1361-9.

22 Kotlyar AM, Grechukhina O, Chen A, Popkhadze S, Grimshaw A, Tal O, et al. Vertical transmission of coronavirus disease 2019: a systematic review and meta-analysis. Am J Obstet Gynecol. 2021 Jan;224(1):35-e3.

23 Cribiù FM, Erra R, Pugni L, Rubio-Perez C, Alonso L, Simonetti S, et al. Severe SARSCoV-2 placenta infection can impact neonatal outcome in the absence of vertical transmission. J Clin Invest. 2021 Mar 15;131(6): e145427.

24 Lou-Mercadé AC, Gavín O, Oros D, Paules C, Savirón-Cornudella R, Mateo P, et al. Prevention of thrombosis in pregnant women with suspected SARS-CoV-2 infection: clinical management algorithm. Ultrasound Obstet Gynecol. 2020 Jul;56(1):111-2.

25 Gao Z, Xu Y, Sun C, Wang X, Guo Y, Qiu S, et al. A systematic review of asymptomatic infections with COVID-19. J Microbiol Immunol Infect. 2021;54(1):12-6.

26 Oros D, Strunk M, Breton P, Paules C, Benito $\mathrm{R}$, Moreno E, et al. Altered gene expression in human placenta after suspected preterm labour. Placenta. 2017;55:21-8.

27 Tom MR, Mina MJ. To interpret the SARSCoV-2 test, consider the cycle threshold value. Clin Infect Dis. 2020 Nov 19;71(16):22524.

28 Brown MA, Magee LA, Kenny LC, Karumanchi SA, McCarthy FP, Saito S, et al. Hypertensive disorders of pregnancy: ISSHP classification, diagnosis, and management recommendations for international practice. Hypertension. 2018;72(1):24-43.

29 Serra B, Mendoza M, Scazzocchio E, Meler E, Nolla M, Sabrià E, et al. A new model for screening for early-onset preeclampsia. Am J Obstet Gynecol. 2020 Jun;222(6):608-e18.

30 Shanes ED, Mithal LB, Otero S, Azad HA, Miller ES, Goldstein JA. Placental pathology in COVID-19. Am J Clin Pathol. 2020 Jun 8; 154(1):23-32

31 Prabhu M, Cagino K, Matthews KC, Friedlander RL, Glynn SM, Kubiak JM, et al. Pregnancy and postpartum outcomes in a universally tested population for SARS-CoV-2 in New York city: a prospective cohort study. BJOG. 2020;127(12):1548-56.
32 Penfield CA, Brubaker SG, Limaye MA, Lighter J, Ratner AJ, Thomas KM, et al. Detection of severe acute respiratory syndrome coronavirus 2 in placental and fetal membrane samples. Am J Obstet Gynecol MFM. 2020;2(3):100133.

33 Hosier H, Farhadian SF, Morotti RA, Deshmukh U, Lu-Culligan A, Campbell KH, et al. SARS-CoV-2 infection of the placenta. J Clin Invest. 2020;130(9):4947-53.

34 Lu-Culligan A, Chavan AR, Vijayakumar P, Irshaid L, Courchaine EM, Milano KM, et al. Maternal respiratory SARS-CoV-2 infection in pregnancy is associated with a robust inflammatory response at the maternal-fetal interface. Med. 2021 May 14;2(5):591-610.

35 Brennan LJ, Morton JS, Davidge ST. Vascular dysfunction in preeclampsia. Microcirculation. 2014 Jan;21(1):4-14.

36 Levy A, Yagil Y, Bursztyn M, Barkalifa R, Scharf S, Yagil C. ACE2 expression and activity are enhanced during pregnancy. Am J Physiol Regul Integr Comp Physiol. 2008 Dec; 295(6):R1953-61.

37 Li M, Chen L, Zhang J, Xiong C, Li X. The SARS-CoV-2 receptor ACE2 expression of maternal-fetal interface and fetal organs by single-cell transcriptome study. PLOS One. 2020;15(4):e0230295.

38 Meijer WJ, van Noortwijk AG, Bruinse HW, Wensing AM. Influenza virus infection in pregnancy: a review. Acta Obstet Gynecol Scand. 2015 Aug;94(8):797-819.

39 Olgun NS. Viral infections in pregnancy: a focus on ebola virus. Curr Pharm Des. 2018; 24(9):993-8.

40 Allotey J, Stallings E, Bonet M, Yap M, Chatterjee $\mathrm{S}$, Kew T, et al. Clinical manifestations, risk factors, and maternal and perinatal outcomes of coronavirus disease 2019 in pregnancy: living systematic review and metaanalysis. BMJ. 2020;370:m3320.

41 Zambrano LD, Ellington S, Strid P, Galang RR, Oduyebo T, Tong VT, et al. COVID-19 response pregnancy and infant linked outcomes team. Update: characteristics of symptomatic women of reproductive age with laboratory-confirmed SARS-CoV-2 infection by pregnancy status - United States, January 22-October 3, 2020. MMWR Morb Mortal Wkly Rep. 2020 Nov 6;69(44):1641-7.

42 Patberg ET, Adams T, Rekawek P, Vahanian SA, Akerman M, Hernandez A, et al. Coronavirus disease 2019 infection and placental histopathology in women delivering at term. Am J Obstet Gynecol. 2021 Apr;224(4):382-e18.

43 Matute J, Finander B, Pepin D, Ai X, Smith N, $\mathrm{Li}$ J, et al. Single-cell immunophenotyping of the fetal immune response to maternal SARS$\mathrm{CoV}-2$ infection in late gestation. Res Sq [Preprint]. 2021;rs.3.rs-311000.
SARS-CoV-2 Viral Load in the Placenta of Patients with Hypertensive Disorders
Fetal Diagn Ther 2021;48:801-811 